\title{
Quality Tracing and Control Information System for Extended Enterprise
}

\author{
Liping Zhao, Damin Xu, Yiyong Yao and Yongtao Qin \\ State Key Laboratory for Manufacturing Systems Engineering, Xi'an JiaoTong University, \\ Xi'an 710049, P. R. China \\ School of Mechanical Engineering, Xi' an JiaoTong University, Xi' an 710049, P. R. China \\ lipingzh@mail.xjtu.edu.cn daminxu@gmail.com \\ yyyao@mail.xjtu.edu.cn qinyt23419@126.com
}

\begin{abstract}
With economies tending towards global uniformity, the traditional quality control in a single enterprise has to be innovated. Core competencies or leadership capabilities in quality control have to be strengthened by the complementary capabilities of partners for extended enterprise (E2). So how to establish an economic and effective quality tracing and control information system to trace and control quality of inter-enterprise has becoming one of the studying hotspots currently. In this paper, a quality tracing and control information system for extended enterprise (E2-QTCIS) is explored based on $\mathrm{J} 2 \mathrm{EE}$, and the quality information model of $\mathrm{E} 2$ by using product $\mathrm{BOM}$ and template technology is established. On this basis, the workflow model of quality tracing and control process and the workflow execution logic of tracing and control quality information nodes are constructed. Finally, a four-layer architecture of E2-QTCIS with network transparency and easy expandability is established. E2-QTCIS can capture all quality information quickly, accurately and wholly, and realize quality tracing and control of E2 dynamically.
\end{abstract}

Keywords: Extended enterprise, Information system, Quality control, Dynamical tracing, Work flow

\section{INTRODUCTION}

Under the background of globalization manufacture, enterprises extended their borderlines and form network-featured extended enterprise, which unites core suppliers and customers [1-2]. For extended enterprise and its product collaboration manufacturing, how to establish an economic and effective quality tracing and control information system to trace and control quality of enterprises is one of hotspots of current research.

In theory and practice, the current quality information tracing and control systems with the assumption of organization borderline pay their emphases on an independent enterprise and its product quality [3]. These quality control mode and theory can not be adaptive to network-featured extended enterprise consisting of member enterprises. It needs to be established a quality information system to support collaboration of member enterprises distributed in different regions to assure the efficiency and correctness of collaboration [4-5]. 
Aiming at above demands, quality information model of extended enterprise by using product BOM and template technology is established and workflow model of quality tracing and control process is constructed. The quality tracing and control of enterprises is realized by using tracing and control of quality information nodes. Finally, the architecture of quality tracing and control information system for extended enterprise (E2-QTCIS) is proposed based on J2EE.

\section{QUALITY INFORMATION MODEL OF E2 BASED ON BOM AND TEMPLATE}

Quality information is the information that is concerned with quality and happens in extended enterprise. It exists in data, chart, report and command, and reflects every aspect of quality activities. For the quality information of E2 is complicated and dispersive, it is necessary to establish a reasonable and unified quality information model to organize and manage quality information of E2 for quality tracing and control.

Together with layered and fractal features and corresponding BOM tree of manufacturing product (Figure.1a and Figure.1b), this paper constructs a quality information model based on BOM and template (Figure.1c) [6-8]. In order to clearly describe the relation of quality information, quality information is firstly classified into total product layer, sub-product layer, assembly layer and part layer. And then, some quality information nodes are set in every layer and every quality information node corresponds to a quality information template. Aiming at special quality information node, quality information template encapsulates its all quality information.

The concrete content encapsulated in quality information template can be found from enlarged figure of quality information node. The data layer consists of various quality data and document file. The data object encapsulates data of data layer and adds context information, such as data edition information and state information, to ensure right and integrate application of quality data, which embodies citation of quality data in data layer. Business object layer embodies that different businesses need different application requirements of quality information. According to different application requirements, quality information is abstracted and classified in different layer and different business objects are formed in logic. Business object attributes reflects the layered categorization of quality information aiming at different business requirements. Underplayed business object is obtained from attributes of superlayered business object by further limitation. Categorization, organization and structure of quality information are main content of business object layer.

As shown in Figure.1, quality information model embodies relation of product quality information tracing control object. For product object oriented extended enterprise, quality information template is based on product structure and extends with product structure decomposition. With product evolution, the tracing of quality information is realized by using quality information template recording quality information. 

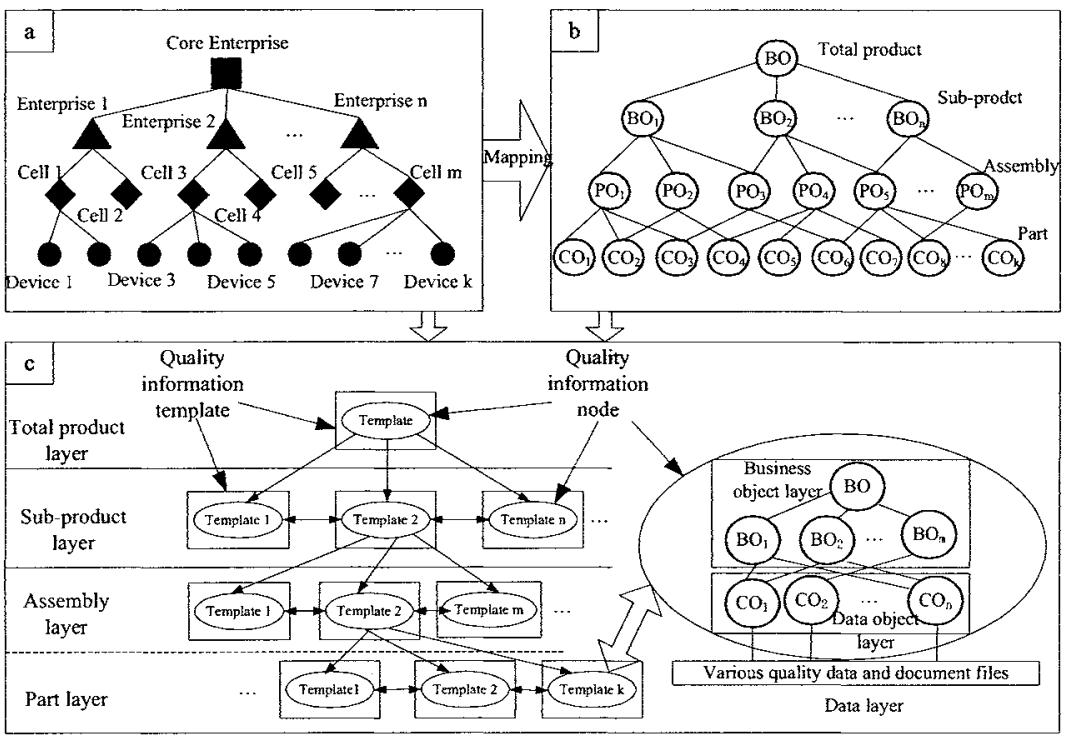

Figure 1. Quality Information System Based on BOM and Template

\section{QUALITY TRACING AND CONTROL WORKFLOW MODEL OF E2}

Quality tracing and control among E2 is more complicated than internal individual enterprise, but there is a close similarity work process of inter-enterprise, so workflow technology is introduced to quality tracing and control process among extended enterprise in this paper [7][9-12].

\section{I Quality Tracing and Control Workflow Process Model of E2}

As it is readable, activity network chart based on directed-graph theory is adopted to construct the process model of quality tracing and control. One business process is composed of one graph in the process model based on activity network chart. Nodes in the graph describe the procedures and assignments, connected arcs between nodes describe the quality control flow and information flow in the process.

The construction theory of process model based on directed graph. By dint of directed graph, we construct a tracing process as five-tuple array:

$$
G=\{s, A N, L N, e, L\}
$$


Where, $S$ is the beginning element of process. $A N=\{M A \cup A A \cup S P\}, M A$ is the nodes aggregation of artificial activity, $A A$ is the nodes aggregation of auto activity, $S P$ is the nodes aggregation of sub-process. $L N=\{O R \cup A N D \cup X O R\}$ is the nodes aggregation of logical activity. $O R$ expresses or logic nodes, $A N D$ expresses and logic nodes, $X O R$ expresses exclusive-or logic nodes. $e$ is close element of process. $L=\left\{l_{1}, l_{2}, \cdots l_{r}\right\}$ is the aggregation of connected arcs. $l_{j}=\left(m_{i}, m_{k}\right)$ expresses the connected arc from $m_{i}$ to $m_{k}$.

There is only a unique starting node $S$ and a close node $e$ at least.

Tracing and control workflow process model According to FRACAS(Failure Reporting, Analysis and Corrective Action System)process, the quality tracing and control process in extended enterprises could be divided into $\mathbf{n}$ paratactic child processes, each child process is a self-closed system, and has its corresponding executive staffs and managers in implementation, it is connected with quality problem report and quality information template between child process. The dashed line connection of final step in each process stands for a new tracing process, as shown in Figure 2.

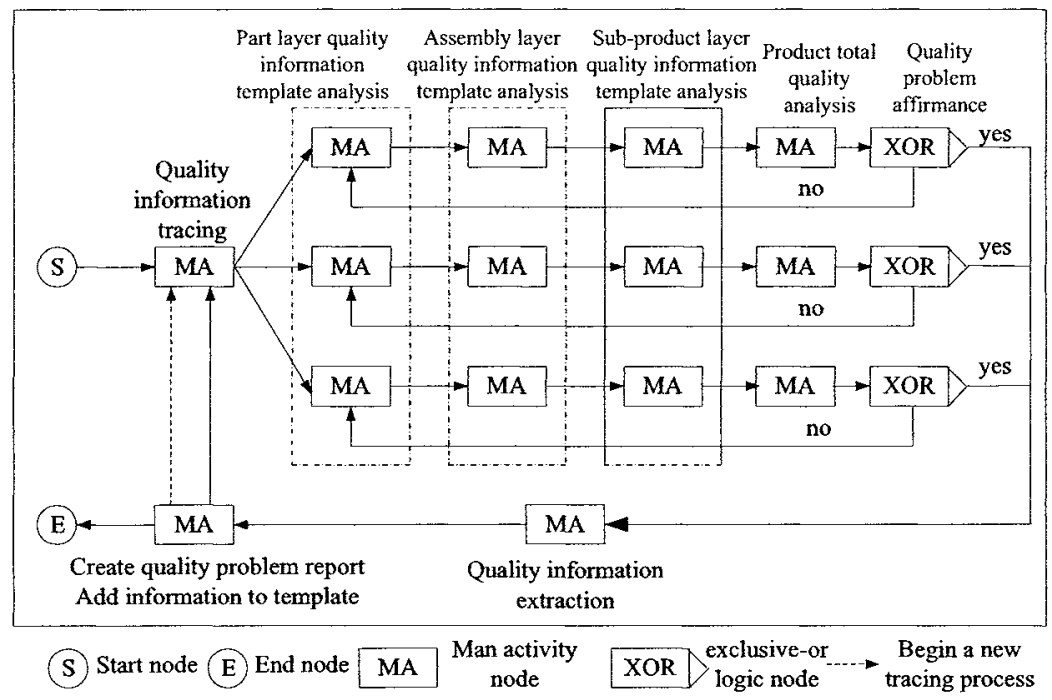

Figure 2. Quality Tracing and Control Workflow Model

Combined process model construct theory of digraph, the process model of quality tracing and control workflow is constructed and expressed as

$$
\mathrm{P}=\left\{s_{q c}, A N_{q c}, L N_{q c}, e_{q c}, L_{q c}\right\}
$$

Where, subscript $q c$ expresses "quality control". 
$s_{q c}$ is start element of process. There is its own corresponding starting element in every tracing process, and each process has only a unique starting node.

$e_{q c}$ is close element of process. In the process of quality tracing, the close nodes of the rest processes are also starting nodes for the next processes besides the first close node $e_{q c}^{1}$, i.e. $e_{q c}^{m}=s_{q c}^{m+1}$, express a new tracing and control process.

$A N_{q c}=\left\{M A_{q c}\right\}$ is the activity nodes aggregation of tracing process. Of which, $M A_{q c}$ is the nodes aggregation of artificial activity, $M A_{q c} \subseteq N_{q c}, M A_{q c}=$ \{discover problems, checking problems $\cdots$ \} etc. $M A_{q c}$ is primarily responsible for the collection of relevant information collection and testing, and makes decision about information.

$L N_{q c}=\left\{O R_{q c} \cup A N D_{q c} \cup X O R_{q c}\right\}$ is the nodes aggregation of logic activity. The logic nodes are only involved with exclusive-or logic $X O R_{q c}$ in tracing process, as $X O R_{q c}=L N_{q c}, X O R_{q c}$ is in charge of gaining result of knowledge judgment, and choosing different paths in tracing process.

$L_{q c}=\left\{l_{1}, l_{2}, l_{3}, \cdots, l_{i}\right\}$ is the aggregation of connected arcs in tracing process. Of which, $l_{i}=\left\{M A_{j}, M A_{k}\right\}$ expresses the connected arcs from node $M A_{j}$ to $M A_{k}$. For example, $l_{2}=\left\{M A_{1}, M A_{2}\right\}$ expresses the connected arcs from artificial activity node $M A_{1}$ "quality information tracing" to node $M A_{2}$ "quality information node of enterprises layer".

Figure 2 fully reflects the layer property and coordination in the quality tracing process. The whole tracing and control process is carried out from the coordination between the nodes of artificial activity and nodes of logic activity, and the node between each other are contacted though quality information templates, the quality information templates could be divided into part level, component level, and child product level according to the level relationship of executing. The necessary condition of the next node's executing is the information and knowledge provided by the anterior node. When several tracing processes are executing together, workflow is used to monitor working state of each nodes, and correspond job schedule of each node and child process, in order to assure several quality tracing and control process could be executed betimes and effectively. The quality problem information of every level will be picked up at the end, and collected to generate a quality problem record; meanwhile, the quality information template is modified, as a preparation for the next quality tracing and control process.

\subsection{Realization of Quality Tracing and Control Model}

In this paper we establishes a quality tracing and control model by referring to directed graph theory of process construction and FRACAS flow, which combines the characteristics and demand of inter-extended enterprise. The logic realization framework of quality tracing and control model is shown in Figure3. 
First, all the quality information is classed into four layers that are total product layer, sub-product layer, assembly layer and part layer by product BOM. In each layer, some quality information nodes and corresponding quality information templates are set up, various kinds of quality information is encapsulated in quality information template. The quality information model is built on BOM and quality information templates. For each information node is accompanied with activity, we define the nodes accompanied with activity as quality tracing and control nodes of workflow model, at the same time, establish the relation of nodes by the constraint relation of organization and resource. The quality tracing and control of interextended enterprise is realized by tracing and control of nodes.

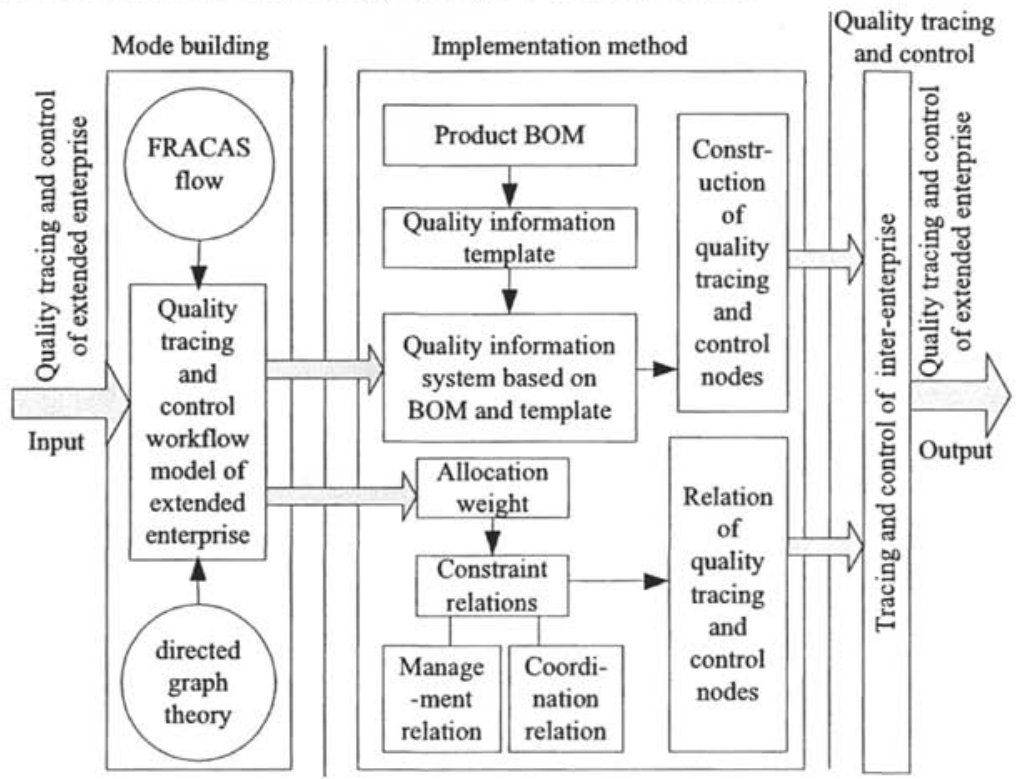

Figure 3. Logic Realization Framework of Quality Tracing and Control Workflow Model

\section{E2-QTCIS ARCHITECTURE}

E2-QTCIS is designed as B/S pattern. As shown in Figure4, system implementation adopts J2EE frame and comprises of four layers in the architecture, such as user access layer, application implement layer, application layer and network support layer. JSP and Servlets technology provides Web service [13-16].

User access layer This layer is mostly used for the interaction of user and system. Workers, clients and suppliers visit information system via Web browser. System access layer visits Web service via HTTP and then returns to interface which generated by HTML or XML. 
Application execution layer Resources distributing consists with organization structure of extended enterprise. It can be classified into enterprise-level resources, cell-level resources and device-level resources. Static information and dynamic information generated in function of various resources are stored in the database of data layer.

Application layer This layer consists of data layer and function layer. Data layer includes all information which supports the operation of E2-QTCIS, such as resource information, quality information etc. Function layer integrates a series of 0application function modules which are realized by quality information management and controller based on product BOM. The function modules of E2- QTCIS are shown in Figure4, including basic function module, core function module and some other extended function modules. Core function module includes information tracing, control and feed back, quality guarantee tools, quality information cooperation tools and quality control process and etc.

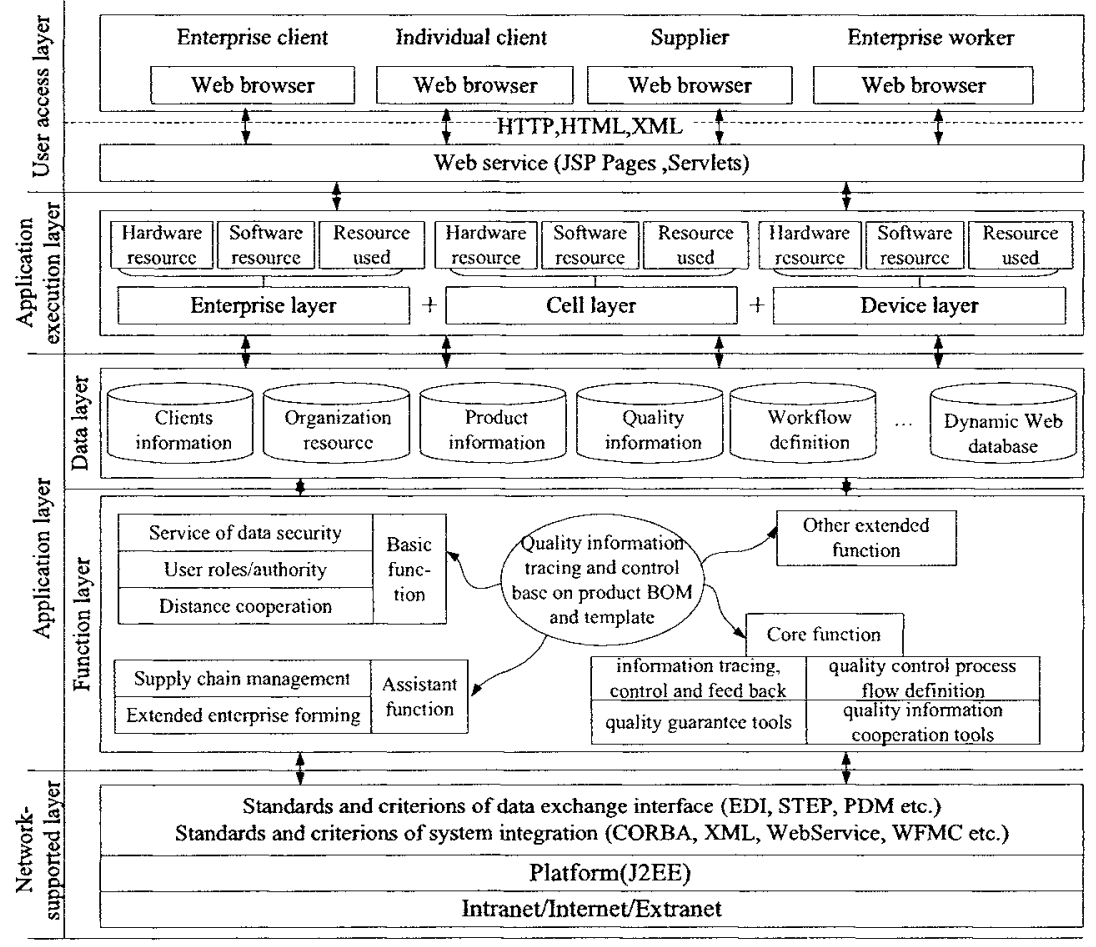

Figure 4. Architecture of E2-QTCIS

Network-supported layer On J2EE platform, we adopt Web Service and CORBA technology to construct system on Intranet/Intemet/Extranet, which make system possess network transparency and easy to maintain and also easy to extension. 


\section{CONCLUSIONS}

An E2-QTCIS based on J2EE is presented in this paper, the research has exposed the following results:

1. The quality information model based on BOM and template lays the foundation for the following quality tracing and control of inter-enterprise. Together with layered and fractal features and corresponding BOM tree of manufacturing product, the relation of quality information is described clearly, at the same time, complicated and dispersive quality information is organized and managed in a reasonable and unified quality information model. All of this provides convenience for the realization of quality tracing and control workflow model.

2. With the aid of quality information model, Workflow Model can trace and control working state of each node each layer, improves cooperativity and work efficiency. Quality tracing and control among E2 is more complicated than internal individual enterprise, but there is a close similarity work process of inter-enterprise, so workflow technology is introduced to quality tracing and control process among extended enterprise in this paper.

3. A four-layer architecture of E2-QTCIS with network transparency and easy expandability is established. E2-QTCIS integrates quality information and function and process of inner-enterprise and inter-enterprise, can realize the goal of quality tracing and control by acquiring various quality information fast and accurately.

4. This research offers theoretical foundation to simplify the development of QTCIS. It also provides a new idea and a resolution for quality tracing and control of interenterprise, simultaneously, has active meaning for practical application of network manufacturing. The development of prototype system remains for further research.

\section{ACKNOWLEDGEMENTS}

This work was supported by the National High-Tech. R\&D Program for contemporary manufacturing integrated technology, China (No. 2006AA04Z149).

\section{REFERENCES}

1. X. Tang, Quality Management Based on Modern Manufacturing (Science Press: Beijing, 2004).

2. Q. Lv and X. Tang, Quality Management Information System for Cooperative Manufacturing, Journal of Beijing University of Aeronautics and Astronautics. Volume 30, Number 4, pp.316-320, (2004).

3. Z. He, Q. Cui, and H. He, Development of Integrated Quality Information System for Continuous Improvement, in Proc. of IEEE International Conference on Management of Innovation and Technology (ICMIT) (New York, NY, 2006), pp.830-833.

4. J. Giovani, D. Silveira, and R. Cagliano, The relationship between interorganizational information systems and operations performance, International Journal of Operations \& Production Management. Volume 26, Number 3, pp.232-253, (2006). 
5. J. Roger, S. Pokharel, A. Kumar, and L. Zhang, Development of an online quality information system for e-manufacturing, Journal of Manufacturing Technology Management. Volume 18, Number 1, pp.36-53, (2007).

6. H. Sun and P. Jiang, Template-Based Manufacturing Information Tracking Method for Extended Enterprise, Journal of Xi'an Jiaotong University. Volume 40, Number 1, pp.3639, (2006).

7. H. Dong, D. Liu, Y. Zhao, and Y. Chen, Monitoring Approach of Schedule Progress in Fractal Web-based Cooperative Manufacturing System, Computer Integrated Manufacturing System. Volume 10, Number 4, pp.779-783, (2004).

8. J. Ramanathan, Fractal architecture for the adaptive complex enterprise, Adaptive complex enterprises. Volume 48, Number 5, pp.1089-1108, (2005).

9. L. Zhao and M. Wang, Quality Control System Model Based on the Web Workflow, Computer Integrated Manufacturing System. Volume 10, Number 5, pp.502-507, (2004).

10. W. Wei, L. Zhao, and Y. Yao, Collaborative Quality Tracking Model Based on Workflow, Computer Integrated Manufacturing System. Volume 12, Number 10, pp.1586-1590, (2006).

11. D. Sun, X. Xiong and L. Ruan, Workflow-driven Collaborative Session Management in Product Lifecycle Management via Internet, in Proc. of IEEE International Engineering Management Conference (IEMC), Volume 3, Innovation and Entrepreneurship for Sustainable Development (Singapore, 2004), pp.1146-1150.

12. P. Senkur and I. Toroslu, An Architecture for Workflow Scheduling under Resource Allocation Constrains, Information System. Volume 30, pp.399-422, (2005).

13. X. Liu, C. Liu, and Y. Ma, Research on Dynamic Quality Control System Based on MES, Computer Integrated Manufacturing System. Volume 11, Number 1, pp.133-137, (2005).

14. K. Tam and R. Tsang, Timeliness and transaction management in extended enterprises, International Journal of Business Information Systems. Volume 2, Number 4, pp.392$412,(2007)$.

15. X. Zhu and H. Wang, An Integrated Information System for Financial Investment, in the International Federation for Information Processing (IFIP), Volume 205, Research and Practical Issues of Enterprise Information System, eds. A.M. Tjoa and L. Xu (Springer: Boston, MA, 2006), pp.449-456.

16. E. Neaga and J. Harding, An enterprise modeling and integration framework based on knowledge discovery and data mining, International Joumal of Production Research. Volume 43, Number 6, pp.1089-1108, (2005). 